\title{
Quadratic solitons as nonlocal solitons
}

\section{Nikolov, Nikola Ivanov; Neshev, D.; Bang, Ole; Królikowski, W.}

\section{Published in:}

Physical Review E. Statistical, Nonlinear, and Soft Matter Physics

Link to article, DOI:

10.1103/PhysRevE.68.036614

Publication date:

2003

\section{Document Version}

Publisher's PDF, also known as Version of record

Link back to DTU Orbit

\section{Citation (APA):}

Nikolov, N. I., Neshev, D., Bang, O., \& Królikowski, W. (2003). Quadratic solitons as nonlocal solitons. Physical Review E. Statistical, Nonlinear, and Soft Matter Physics, 68(3), 366141-366145. [036614].

https://doi.org/10.1103/PhysRevE.68.036614

\section{General rights}

Copyright and moral rights for the publications made accessible in the public portal are retained by the authors and/or other copyright owners and it is a condition of accessing publications that users recognise and abide by the legal requirements associated with these rights.

- Users may download and print one copy of any publication from the public portal for the purpose of private study or research.

- You may not further distribute the material or use it for any profit-making activity or commercial gain

- You may freely distribute the URL identifying the publication in the public portal 


\title{
Quadratic solitons as nonlocal solitons
}

\author{
Nikola I. Nikolov \\ Informatics and Mathematical Modelling, Technical University of Denmark, 2800 Kongens Lyngby, Denmark \\ and Department of Optics and Fluid Dynamics, Risb National Laboratory, OFD-128, P.O. Box 49, 4000 Roskilde, Denmark \\ Dragomir Neshev \\ Nonlinear Physics Group and ARC Centre of Excellence for Ultrahigh-Bandwidth Devices for Optical Systems, \\ Research School of Physical Sciences and Engineering, Australian National University, \\ Canberra, Australian Capital Territory 0200, Australia \\ Ole Bang \\ Research Center COM and Department of Informatics and Mathematical Modelling, Technical University of Denmark, \\ 2800 Kongens Lyngby, Denmark \\ Wiesław Z. Królikowski \\ Laser Physics Centre and ARC Centre of Excellence for Ultrahigh-Bandwidth Devices for Optical Systems, \\ Research School of Physical Sciences and Engineering, Australian National University, \\ Canberra, Australian Capital Territory 0200, Australia \\ (Received 26 February 2003; published 26 September 2003; published 29 September 2003)
}

\begin{abstract}
We show that quadratic solitons are equivalent to solitons of a nonlocal Kerr medium. This provides new physical insight into the properties of quadratic solitons, often believed to be equivalent to solitons of an effective saturable Kerr medium. The nonlocal analogy also allows for analytical solutions and the prediction of bound states of quadratic solitons.
\end{abstract}

DOI: 10.1103/PhysRevE.68.036614

PACS number(s): 42.65.Tg, 42.65.Ky, 42.65.Sf, 05.45.Yv

\section{INTRODUCTION}

Quadratic nonlinear (or $\chi^{(2)}$ ) materials have a strong and fast electronic nonlinearity, which makes them excellent materials for the study of nonlinear effects, such as solitons [1]. The main properties of quadratic solitons are well known [2] and both $(1+1)$-dimensional [3] and $(2+1)$-dimensional [4] bright spatial solitons have been observed experimentally. Unlike conventional solitons, which form due to a selfinduced refractive index change, the formation of quadratic solitons does not involve any change of the refractive index. Thus the underlying physics of quadratic solitons is often obscured by the mathematical model. Only recently Assanto and Stegeman used the concepts of the cascading phase shift and parametric gain to give an intuitive interpretation of effects, such as self-focusing, defocusing, and soliton formation in $\chi^{(2)}$ materials [5].

Nevertheless certain features of quadratic solitons, such as formation of bound states, are still without a physical interpretation. The nice simple phase-shift approach of Assanto and Stegeman [5] predicts, e.g., that two dark solitons and two out-of-phase bright solitons will always repel and thus can never form a bound state, whereas it is known that such bound states of quadratic solitons do exist [6].

Here we use the analogy between parametric interaction and nonlocality and present a physically intuitive nonlocal theory, which is exact in predicting the profiles of stationary quadratic solitons and which provides a simple physical explanation for their properties including formation of bound states.

The nonlocal analogy was applied recently by Shadrivov and Zharov to find approximate bright quadratic soliton solutions [7]. The formal equivalence of bright solitons in nonlocal liquid crystals and parametric solitons was also discussed recently by Conti, Peccianti, and Assanto [8]. However, the nonlocal concept was not fully exploited in Refs. [7,8] to give a broad physical picture in the whole regime of existence and discuss, e.g., dark solitons and bound states of out-of-phase bright solitons.

We do that here and we go one step further in showing how a simple phase-sensitive nonlocal model provides a better description of the dynamics in $\chi^{(2)}$ materials than the nonlinear Schrödinger (NLS) equation obtained in the cascading limit.

We consider a fundamental wave (FW) and its second harmonic $(\mathrm{SH})$ propagating along the $z$ direction in a lossless quadratic nonlinear medium under conditions for type I phase matching. The normalized dynamical equations for the slowly varying envelopes $E_{1,2}(x, z)$ are then [9]

$$
\begin{gathered}
i \partial_{z} E_{1}+d_{1} \partial_{x}^{2} E_{1}+E_{1}^{*} E_{2} \exp (-i \beta z)=0, \\
i \partial_{z} E_{2}+d_{2} \partial_{x}^{2} E_{2}+E_{1}^{2} \exp (i \beta z)=0 .
\end{gathered}
$$

In the spatial domain $d_{1} \approx 2 d_{2}, d_{j}>0$, and the coordinate $x$ represents a transverse spatial direction. The term $\partial_{x}^{2} E_{j}$ $=\partial^{2} E_{j} / \partial x^{2}$ then represents beam diffraction. In the temporal domain $d_{j}$ is arbitrary and $x$ represents time. In this case $\partial_{x}^{2} E_{j}$ represents pulse dispersion. The parameter $\beta$ is the normalized phase mismatch and $j=1,2$. 


\section{GENERAL NONLOCAL MODEL}

Physical insight into the properties of Eqs. (1) and (2) may be obtained from the cascading limit, in which the phase mismatch is large, $\beta^{-1} \rightarrow 0$. Writing $E_{2}=e_{2} \exp (i \beta z)$ and assuming slow variation of $e_{2}(x, z)$ gives the NLS equation

$$
i \partial_{z} E_{1}+d_{1} \partial_{x}^{2} E_{1}+\beta^{-1}\left|E_{1}\right|^{2} E_{1}=0
$$

in which the local Kerr nonlinearity is due to the coupling to the SH field $e_{2}=E_{1}^{2} / \beta$. The SH is thus slaved to the FW and the widths of the SH and FW are fixed. The sign of the mismatch $\beta$ determines whether the effective Kerr nonlinearity is focusing or defocusing and thus the cascading limit predicts that bright and dark quadratic solitons exist for $\beta d_{1}>0$ and $\beta d_{1}<0$, respectively.

However, even for stationary solutions the NLS equation is inaccurate, since the term $\partial_{x}^{2} E_{2}$ is neglected. Thus it predicts, e.g., modulational stability of dark quadratic solitons for all values of $d_{2}$, whereas this is known to depend on the value of $d_{2}$ [6]. It further predicts that in higher dimensions bright solitons are unstable and will either spread out or collapse [10], whereas it is known that stable quadratic solitons exist in all dimensions and that collapse cannot occur in the $\chi^{(2)}$ system (1) and (2) $[11,12]$. The stabilizing effect of the $\chi^{(2)}$ nonlinearity is often described as being due to saturation of the effective Kerr nonlinearity $[5,11,13]$. We show below that the nonlinearity is in fact nonlocal.

To obtain a more accurate model than that given by the cascading limit we assume a slow variation of the $\mathrm{SH}$ field $e_{2}(x, z)$ in the propagation direction only. Thus, neglecting only $\partial_{z} e_{2}$, we solve Eq. (2) exactly using Fourier transformation and the convolution theorem, treating $E_{1}^{2}$ as a function. The $\mathrm{SH}$ is still expressed in terms of the FW, but now the relation has the form of a convolution, leading to the nonlocal equation for the FW:

$$
\begin{gathered}
i \partial_{z} E_{1}+d_{1} \partial_{x}^{2} E_{1}+\beta^{-1} N\left(E_{1}^{2}\right) E_{1}^{*}=0, \\
N\left(E_{1}^{2}\right)=\int_{-\infty}^{\infty} R(x-\xi) E_{1}^{2}(\xi, z) d \xi
\end{gathered}
$$

with $E_{2}=\beta^{-1} N \exp (i \beta z)$. Equations (4) and (5) clearly show that the interaction between the $\mathrm{FW}$ and the $\mathrm{SH}$ is equivalent to the propagation of a $\mathrm{FW}$ in a medium with a nonlocal nonlinearity. In the Fourier domain (denoted with tilde) the response function $R(x)$ is a Lorentzian $\widetilde{R}(k)=1 /(1$ $+s \sigma^{2} k^{2}$ ), where $\sigma=\left|d_{2} / \beta\right|^{1 / 2}$ represents the degree of nonlocality and $s=\operatorname{sgn}\left(d_{2} \beta\right)$. Both Eqs. (1) and (2) and (4) and (5) are trivially extended to include more transverse dimensions.

For $s=+1$, where the $\chi^{(2)}$ system (1) and (2) has a family of bright (for $d_{1}>0$ ) and dark (for $d_{1}<0$ ) soliton solutions [6], $\widetilde{R}(k)$ is positive definite and localized, giving

$$
R(x)=(2 \sigma)^{-1} \exp (-|x| / \sigma)
$$

The cascading limit $\beta^{-1} \rightarrow 0$ is now seen to correspond to the local limit $\sigma \rightarrow 0$, in which the response function becomes a $\delta$ function, $R(x) \rightarrow \delta(x)$.

With the nonlocal analogy one can assign simple physically intuitive pictures to several important cases. When the mismatch $|\beta|$ is large Eqs. (4) and (5) reduce to the so-called weakly nonlocal equation with $\sigma \ll 1$ [14]. Similarly the nearly phase-matched limit when $\beta \approx 0$ corresponds to the strongly nonlocal limit with $\sigma \gg 1$, when Eqs. (4) and (5) become effectively linear $[15,16]$.

For $s=-1, \widetilde{R}(k)$ has poles on the real axis and the response function becomes oscillatory in nature with the Cauchy principal value

$$
R(x)=(2 \sigma)^{-1} \sin (|x| / \sigma) .
$$

In this case the propagation of solitons has a close analogy with the evolution of a particle in a nonlinear oscillatory potential. In fact, it is possible to show that the oscillatory response function explains the fact that dark and bright quadratic solitons radiate linear waves for $s=-1$ [6].

Equations (4) and (5) show the important result that in contrast to the conventional nonlocal NLS equation, describing materials with a thermal [18] or diffusion [19] type nonlinearity, liquid crystals [20], and photorefractive crystals [21], the nonlocal response of the $\chi^{(2)}$ system depends on the square of the FW, not its intensity. Thus the phase of the FW enters into the picture and one cannot directly transfer the known nonlocal stability results for plane waves $[15,17]$ and solitons $[14,16]$.

However, the simple nonlocal model (4) and (5) is indeed an improved model of quadratic nonlinear materials, as compared with the even simpler NLS equation obtained in the cascading limit. Thus the nonlocal model correctly shows that the properties of quadratic solitons depends sensitively on the parameter $d_{2}$. For symmetric response functions, $R(x)=R(-x)$, the nonlocal model in arbitrary dimension conserves both power and Hamiltonian and it is straightforward to carry out the same analysis as for the conventional nonlocal NLS equation [16] and show that the Hamiltonian for the system (4) and (5) is also bounded from below. Thus the nonlocal model (4) and (5) also correctly predicts that collapse cannot occur in the $\chi^{(2)}$ system. Furthermore, for stationary fields $E_{1}(x, z)=E_{1}(x)$ the nonlocal model (4) and (5) represents an exact model for $\chi^{(2)}$ materials.

\section{NONLOCAL QUADRATIC SOLITONS}

The properties of nonlocal solitons in terms of profiles thus directly apply to quadratic solitons. Consider stationary solutions to Eqs. (1) and (2) in the form

$$
\begin{gathered}
E_{1}(x, z)=a_{1} \phi_{1}(\tau) \exp (i \lambda z), \\
E_{2}(x, z)=a_{2} \phi_{2}(\tau) \exp (i 2 \lambda z+i \beta z),
\end{gathered}
$$

where the profile $\phi_{j}(\tau)$ is real, with $\tau=x\left|\lambda / d_{1}\right|^{1 / 2}, a_{1}^{2}$ $=\lambda^{2}\left|d_{2} /\left(2 d_{1}\right)\right|$, and $a_{2}=\lambda$. This scaling reduces the number of free parameters to one and transforms Eqs. (1) and (2) into the following system [6]: 


$$
\begin{aligned}
& s_{1} \phi_{1}^{\prime \prime}-\phi_{1}+\phi_{1} \phi_{2}=0, \\
& s_{2} \phi_{2}^{\prime \prime}-\alpha \phi_{2}+\phi_{1}^{2} / 2=0,
\end{aligned}
$$

where $s_{j}=\operatorname{sgn}\left(\lambda d_{j}\right)= \pm 1, \alpha=(2+\beta / \lambda)\left|d_{1} / d_{2}\right|$, and prime denotes differentiation with respect to the argument. The properties of solitons described by Eqs. (10) and (11) are well known [6]. A family of bright (dark) solitons exist for $s_{2}=s_{1}=+1 \quad\left(s_{2}=-s_{1}=+1\right)$ and $\alpha>0$. As discussed above we do not consider the combinations $s_{2}= \pm s_{1}=-1$, for which solitons do not exist in the whole $\alpha$ space.

Equation (11) has the formal solution $\phi_{2}=\gamma N\left(\phi_{1}^{2}\right)$, with $\gamma=1 /(2 \alpha)$ and the nonlocal nonlinearity $N\left(\phi_{1}^{2}\right)$ given by Eq. (5). For $\operatorname{sgn}\left(s_{2} \alpha\right)=+1$ the response function is $R(\tau)$ $=(2 \bar{\sigma})^{-1} \exp (-|\tau| \bar{\sigma})$, with the degree of nonlocality $\bar{\sigma}$ $=|\alpha|^{-1 / 2}$. Inserting the SH into Eq. (10) then gives the exact nonlocal model for the FW in the $\chi^{(2)}$ system (10) and (11):

$$
s_{1} \partial_{\tau}^{2} \phi_{1}-\phi_{1}+\gamma \phi_{1} \int_{-\infty}^{\infty} R(\tau-\xi) \phi_{1}^{2}(\xi) d \xi=0
$$

where $\gamma$ is the strength of the nonlocal nonlinearity. Thus $\chi^{(2)}$ solitons are equivalent to nonlocal solitons.

In the weakly nonlocal case $\bar{\sigma} \ll 1$ (i.e., $|\alpha| \gg 1$ ) the response function $R(\tau)$ is much narrower than the FW intensity $\phi_{1}^{2}$. Taylor expansion of $\phi_{1}^{2}$ under the integral in Eq. (12) gives the weakly nonlocal model [14]

$$
s_{1} \partial_{\tau}^{2} \phi_{1}-\phi_{1}+\gamma\left(\phi_{1}^{2}+\bar{\sigma}^{2} \partial_{\tau}^{2} \phi_{1}^{2}\right) \phi_{1}=0
$$

where $\phi_{2}=\gamma\left(1+\bar{\sigma}^{2} \partial_{\tau}^{2}\right) \phi_{1}^{2}$. This model has exact bright soliton solutions for $s_{2}=s_{1}=+1$ and $\alpha>0$ [14]:

$$
\pm \tau=\tanh ^{-1}(\rho)+2 \bar{\sigma} \tan ^{-1}(2 \bar{\sigma} \rho)
$$

where $\rho^{2}=\left(a_{1}^{2}-\phi_{1}^{2}\right) /\left(a_{1}^{2}+4 \bar{\sigma}^{2} \phi_{1}^{2}\right), \quad a_{1}^{2}=2 / \gamma$ being the maximum intensity of the FW. Exact stationary black soliton solutions exist for $s_{2}=-s_{1}=+1$ [14].

For $|\alpha| \ll 1$ the nonlocality is strong, $\bar{\sigma} \gg 1$, and we can expand the response function $R(\tau)$ in Eq. (12). For bright solitons we then obtain the linear equation for the FW:

$$
s_{1} \partial_{\tau}^{2} \phi_{1}-\phi_{1}+\gamma P_{1} R(\tau) \phi_{1}=0,
$$

where $\phi_{2}=\gamma P_{1} R(\tau)$. In this eigenvalue problem the FW power $P_{1}=\int_{-\infty}^{\infty} \phi_{1}^{2}(\tau) d \tau$ plays the role of the eigenvalue, and bright solitons correspond to the fundamental mode of the waveguide structure one can associate with the exponential response function. For $s_{2}=s_{1}=+1$ and $\alpha>0$, Eq. (15) has exact bright soliton solutions in the form of the Bessel function of the first kind of order $2 \bar{\sigma}$ [22]

$$
\phi_{1}(\tau)=A_{1} J_{2} \bar{\sigma}\left(\bar{\sigma}^{2} \sqrt{2 P_{1} R(\tau)}\right) .
$$

For the single-soliton ground-state solution $P_{1}$ is found as the first zero of the derivative, $J_{2 \bar{\sigma}}^{\prime}\left(\sqrt{\overline{\sigma^{3} P_{1}}}\right)=0$, which assures that $\phi_{1}^{\prime}(0)=0$. The amplitude $A_{1}$ is then found from the definition of $P_{1}$, giving $A_{1}^{2} \approx P_{1} / 2-\left[P_{1} /\left(\pi^{2} \bar{\sigma}\right)\right]^{1 / 2}$.
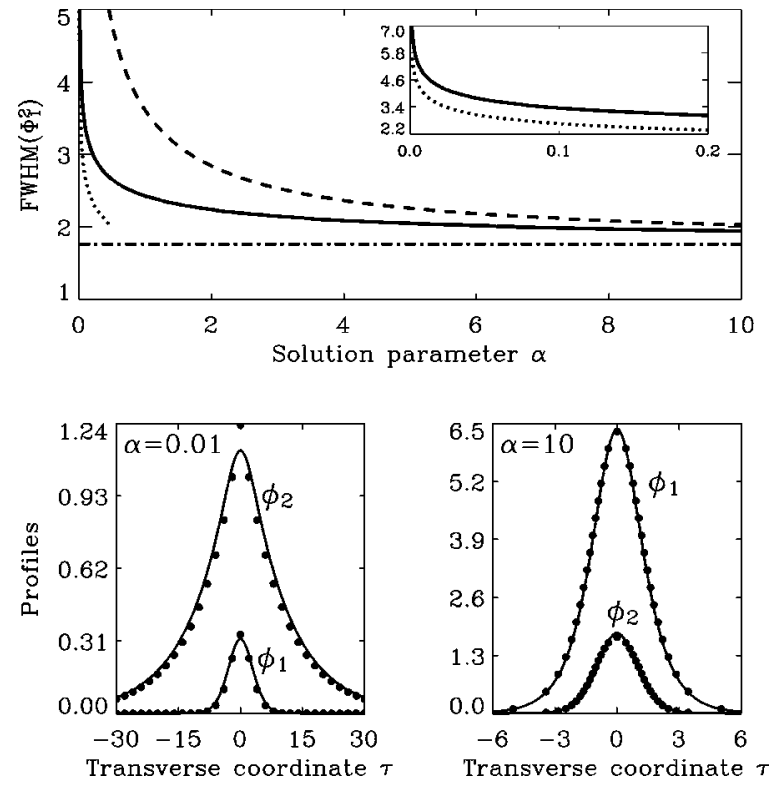

FIG. 1. Top: Numerically found $\operatorname{FWHM}\left(\phi_{1}^{2}\right)$ of bright quadratic solitons vs $\alpha$ (solid), and the weakly nonlocal (dashed), strongly nonlocal (dotted), and cascading limit (chain-dashed) predictions. Bottom: Numerically found profiles (solid) and strongly nonlocal (left, $\alpha=0.01$ ) and weakly nonlocal (right, $\alpha=10$ ) solutions (dots). $s_{2}=s_{1}=+1$.

In Fig. 1 we show the full width at half maximum (FWHM) of the FW intensity $\phi_{1}^{2}$ of bright quadratic solitons versus the phase-mismatch parameter $\alpha$. The analytical solutions obtained using the nonlocal analogy correctly capture the increase of the soliton width with decreasing $\alpha$. The nonlocal model elegantly explains this effect: Because of the convolution in the nonlinearity in Eq. (12) representing a trapping potential or waveguide structure, this potential is always broader than the FW intensity profile itself, leading to its weaker confinement and larger width when the degree of nonlocality increases. The profiles shown in Fig. 1 further illustrate the excellent agreement of the numerical results and approximate nonlocal analytical solutions in both the weakly $(\alpha \gg 1$ ) and the strongly ( $\alpha \ll 1$ ) nonlocal limit.

The linear Eq. (15) describing the strongly nonlocal limit further predicts the existence of multihump bright solitons. Choosing $P_{1}$ as the $N$ th zero of the derivative of the Bessel function, i.e., the $N$ th root in the equation $J_{2}^{\prime}-\left(\sqrt{\bar{\sigma}^{3} P_{1}}\right)=0$, gives solitons with an odd number of humps $(2 N-1)$, as discussed in Ref. [7]. However, this does not exhaust all soliton solutions supported by the model (15). There also exist antisymmetric solitons with an even number of intensity peaks. If the power $P_{1}$ is found as the $N$ th zero of the Bessel function itself, and not its derivative, i.e., as the $N$ th root in the equation $J_{2} \bar{\sigma}\left(\sqrt{\overline{\sigma^{3} P_{1}}}\right)=0$ [so that $\phi_{1}(0)=0$ ], then antisymmetric solitons with an even number of intensity peaks $(2 N)$ exist with the form

$$
\phi_{1}(\tau)=s_{\tau} A_{1} J_{2} \bar{\sigma}\left(\bar{\sigma}^{2} \sqrt{2 P_{1} R(\tau)}\right)
$$

where $s_{\tau}=\operatorname{sgn}(\tau)$. When $P_{1}$, e.g., is fixed by the first zero of $J_{2} \bar{\sigma}$ then solution (17) is a two-peak antisymmetric soliton, 


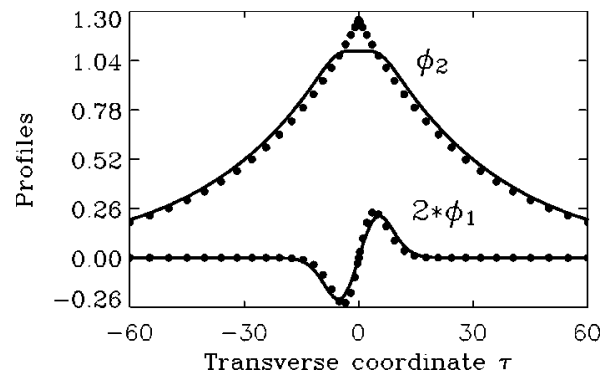

FIG. 2. Numerically found bound state of two out-of-phase bright solitons for $\alpha=0.001$ (solid) and the predicted strongly nonlocal solution (17) (dashed); $s_{2}=s_{1}=+1$.

which can be interpreted as a bound state of two out-of-phase fundamental solitons. In Fig. 2 we have shown the bound state of two out-of-phase fundamental solitons predicted by Eq. (17), and the corresponding numerically found solution for $\alpha=0.001$. We see again that the strongly nonlocal model provides an excellent prediction of this bound state quadratic soliton solution.

In fact, all higher order solitons can be thought of as a bound state of a number of individual solitons. Formation of such bound states follows naturally from the nonlocal character of the nonlinear interaction. Consider two out-of-phase solitons, for which the intensity in the overlapping region is always zero. In local Kerr media the nonlinear change in the refractive index is decreased in the overlap region, as compared to the index change generated by a single soliton. This leads to a mutual repulsion of the solitons. The nonlocality tends to increase the nonlinear change of the refractive index in the overlapping region, and for a sufficiently high degree of nonlocality, the index change may even be higher than that for a soliton in isolation, despite the solitons being out of phase. This creates an attractive force and leads to formation of the bound state.

The linear equation for dark solitons in the strongly nonlocal limit $(\alpha \ll 1)$ has the form

$$
s_{1} \partial_{\tau}^{2} \phi_{1}-\phi_{1}+\gamma\left[A_{1}^{2}-Q_{1} R(\tau)\right] \phi_{1}=0,
$$

where $Q_{1}=\int_{-\infty}^{\infty}\left[A_{1}^{2}-\phi_{1}^{2}(\tau)\right] d \tau$ is the complementary FW power and $\phi_{2}=\gamma\left[A_{1}^{2}-Q_{1} R(\tau)\right]$. For $s_{2}=-s_{1}=+1$ and $\alpha$ $>0$, Eq. (18) has exact dark soliton solutions in the form of the zeroth order Bessel function:

$$
\phi_{1}(\tau)=s_{\tau} \sqrt{2 \alpha} J_{0}\left(\bar{\sigma}^{2} \sqrt{2 Q_{1} R(\tau)}\right) .
$$

For the fundamental single-soliton solution $Q_{1}$ is found as the first zero $J_{0}\left(\sqrt{\bar{\sigma}^{3} Q_{1}}\right)=0$, which gives $Q_{1}=5.8 / \bar{\sigma}^{3}$ and assures that $\phi_{1}(0)=0$. The background amplitude is fixed at $A_{1}^{2}=2 \alpha$. Choosing the $N$ th root gives antisymmetric multihump dark solitons with $2 N-1$ dips in the intensity profile. Choosing instead the $N$ th zero of the derivative, i.e., $J_{0}^{\prime}\left(\sqrt{\overline{\bar{\sigma}^{3} Q_{1}}}\right)=0$ gives symmetric multihump dark solitons with $2 N$ dips in the intensity profile.

In the strongly nonlocal limit the bright soliton is the fundamental mode of the waveguide structure $R(\tau)$ and much narrower than the waveguide. In contrast the dark soliton is a
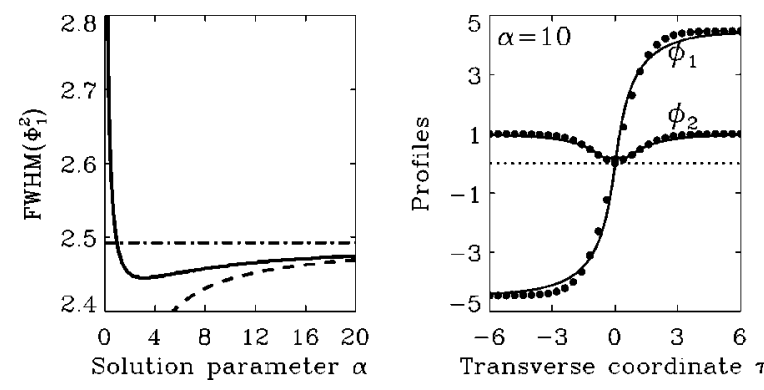

FIG. 3. Left: Numerically found $\operatorname{FWHM}\left(\phi_{1}^{2}\right)$ of dark solitons vs $\alpha$ (solid), and the weakly nonlocal (dashed) and cascading limit (chain-dashed) predictions. Right: Numerically found profiles (dots) and weakly nonlocal solutions (solid) for $\alpha=10 . \phi_{1}^{2}( \pm \infty)=2 \alpha$, $\phi_{2}( \pm \infty)=1$, and $s_{2}=-s_{1}=+1$.

first mode of the waveguide $R(\tau)$ at the cutoff and as such its width is comparable with that of the waveguide. Hence the expansion procedure leading to Eq. (18) is not a good approximation. This is reflected in the fact that the fixed background amplitude $A_{1}$ does not satisfy the self-consistency relation $Q_{1}=\int_{-\infty}^{\infty}\left[A_{1}^{2}-\phi_{1}^{2}(\tau)\right] d \tau$. Nevertheless the strongly nonlocal model is able to predict and physically explain the existence of multihump dark quadratic solitons found earlier [6].

In Fig. 3 we show the full width at half maximum of the FW intensity $\phi_{1}^{2}$ of dark quadratic solitons versus the mismatch parameter $\alpha$. The dark solitons have the constant background $\phi_{1}^{2}( \pm \infty)=2 \alpha, \quad \phi_{2}( \pm \infty)=1$. The analytical weakly nonlocal dark soliton solution exists for $\alpha>2$ and was taken from Ref. [14]. Unlike bright solitons, whose width is a monotonic function of $\alpha$, dark solitons are seen to have a minimum width at $\alpha=\alpha_{0} \approx 3.1$.

Figure 3 confirms that for $\alpha>\alpha_{0}$ the weakly nonlocal model correctly predicts how the soliton width decreases when the mismatch parameter decreases. This, as well as the appearance of the minimum in the soliton width, is again elegantly explained by the nonlocal analogy: Because of the convolution in the nonlinearity in Eq. (12) representing the trapping potential or waveguide structure, the contribution from the constant background tends to contract this potential. This leads to a stronger confinement and thus a smaller width of the soliton. However, this is only true as long as the amplitude of the trapping potential is not affected by nonlocality, as in the weakly nonlocal regime. For a high degree of nonlocality (i.e., smaller value of $\alpha$ ) not only the width of the trapping potential, but also its amplitude is affected. In this regime the nonlocality leads to a drop in the amplitude of the potential, resulting in a weaker confinement and an increase of the soliton width. The profiles shown in Fig. 3 further illustrate the excellent agreement of the numerical results and approximate nonlocal analytical solutions in the weakly nonlocal limit $\alpha \gg 1$.

\section{CONCLUSION}

In conclusion, we have used the analogy between parametric interaction in quadratic media and nonlocal Kerr-type nonlinearities to provide a physically intuitive theory for 
quadratic solitons, which allows for a deeper physical insight into their properties and an exact description of their profiles. Quadratic solitons are characterized by a single solution parameter $\alpha$, which is an effective mismatch parameter depending on both the real phase mismatch and the power. We have shown that the nonlocal theory provides a simple and elegant explanation for how the soliton width depends on the mismatch $\alpha$.

In particular the nonlocal analogy provides simple physical models in both the large mismatch limit $\alpha \gg 1$ and the near cutoff limit $|\alpha| \ll 1$, corresponding to the regimes of weak and strong nonlocality, respectively. Our results show that the weakly nonlocal approximation gives an accurate description of quadratic solitons in a relatively broad range of their existence domain. Also, the simple linear physics of the strongly nonlocal limit has enabled us to find bound states of bright quadratic solitons and explain their formation using the natural concept of the nonlocality-based attraction between out-of-phase constituent solitons.

Finally we have discussed how a simple phase-sensitive nonlocal model provides a better description of the dynamics as compared to the standard NLS equation obtained in the cascading limit.

\section{ACKNOWLEDGMENTS}

The research was supported by the Danish Technical Research Council (Grant No. 26-00-0355) and the Australian Research Council.
[1] For a recent review see G. Stegeman, D.J. Hagan, and L. Torner, 28, 1691 (1996).

[2] For a recent review see A.V. Buryak, P. Di Trapani, D.V. Skryabin, and S. Trillo, Phys. Rep. 370, 63 (2002).

[3] R. Schiek, Y. Baek, and G.I. Stegeman, Phys. Rev. E 53, 1138 (1996).

[4] W.E. Torruellas, Z. Wang, D.J. Hagan, E.W. Van Stryland, G.I. Stegeman, L. Torner, and C.R. Menyuk, Phys. Rev. Lett. 74, 5036 (1995).

[5] G. Assanto and G.I. Stegeman, Opt. Express 10, 388 (2002).

[6] A.V. Buryak and Yu.S. Kivshar, Phys. Lett. A 197, 407 (1995).

[7] I.V. Shadrivov and A.A. Zharov, J. Opt. Soc. Am. B 19, 596 (2002).

[8] C. Conti, M. Peccianti, and G. Assanto, Phys. Rev. Lett. 91, 073901 (2003).

[9] C.R. Menyuk, R. Schiek, and L. Torner, J. Opt. Soc. Am. B 11, 2434 (1994); O. Bang, ibid. 14, 51 (1997).

[10] For a recent review see Yu.S. Kivshar and D.E. Pelinovsky, Phys. Rep. 331, 117 (2000).

[11] L. Bergé, V.K. Mezentsev, J.J. Rasmussen, and J. Wyller, Phys. Rev. A 52, R28 (1995).

[12] L. Bergé, O. Bang, J.J. Rasmussen, and V.K. Mezentsev, Phys.
Rev. E 55, 3555 (1997).

[13] F. Wise and P. Di Trapani, Opt. Photonics News 13, 28 (2002).

[14] W. Krolikowski and O. Bang, Phys. Rev. E 63, 016610 (2001).

[15] W. Krolikowski, O. Bang, J.J. Rasmussen, and J. Wyller, Phys. Rev. E 64, 016612 (2001).

[16] O. Bang, W. Krolikowski, J. Wyller, and J.J. Rasmussen, Phys. Rev. E 66, 046619 (2002).

[17] J. Wyller, W. Krolikowski, O. Bang, and J.J. Rasmussen, Phys. Rev. E 66, 066615 (2002).

[18] A.G. Litvak, V.A. Mironov, G.M. Fraiman, and A.D. Yunakovskii, Fiz. Plazmy 1, 60 (1975) [Sov. J. Plasma Phys. 1, 31 (1975)].

[19] D. Suter and T. Blasberg, Phys. Rev. A 48, 4583 (1993).

[20] D.W. McLaughlin, D.J. Muraki, and M.J. Shelly, Physica D 97, 471 (1996); M. Peccianti, K.A. Brzdakiewicz, and G. Assanto, Opt. Lett. 27, 1460 (2002).

[21] A. Mamaev, A. Zozulya, V.K. Mezentsev, D.Z. Anderson, M. Saffman, Phys. Rev. A 56, R1110 (1997); G.F. Calvo, F. Agullo-Lopez, M. Carrascosa, M. Belic, and W. Krolikowski, Europhys. Lett. 60, 847 (2002).

[22] E.M. Conwell, Appl. Phys. Lett. 23, 328 (1973). 